\title{
CLASSIFICATION OF ESCAPING EXPONENTIAL MAPS
}

\author{
MARKUS FÖRSTER, LASSE REMPE, AND DIERK SCHLEICHER
}

(Communicated by Juha M. Heinonen)

\begin{abstract}
We give a complete classification of the set of parameters $\kappa$ for which the singular value of $E_{\kappa}: z \mapsto \exp (z)+\kappa$ escapes to $\infty$ under iteration. In particular, we show that every path-connected component of this set is a curve to infinity.
\end{abstract}

\section{INTRODUCTION}

In 1777, Euler [E] studied the question for which $a>0$ the limit $a^{a^{a^{*}}}$ exists. Writing $\lambda=\ln a$, this amounts to asking for which $\lambda \in \mathbb{R}$ the iteration

$$
\lambda_{0}:=0, \quad \lambda_{n+1}:=e^{\lambda \cdot \lambda_{n}}
$$

has a limit, which is easily seen to be the case if and only if $-e \leq \lambda \leq 1 / e$. If we instead allow $\lambda$ to vary in the complex plane, the question obtains a much richer structure. It is readily verified that $\left(\lambda_{n}\right)$ has a nontrivial limit if and only if $\lambda$ is of the form $\lambda=\mu e^{-\mu}$, where $|\mu|<1$ or $\mu$ is a root of unity. In addition, there are countably many values of $\lambda$ for which the sequence is eventually constant; a complete classification of these can be found in [LSV, HSS].

This exhausts all cases for which there is a limit in $\mathbb{C}$, but when does $\lambda_{n} \rightarrow \infty$ ? This is certainly the case for all real $\lambda>1 / e$. However, the complete answer is much more interesting:

1.1. Theorem. The set of parameters $\lambda \in \mathbb{C}$ for which $\lambda_{n} \rightarrow \infty$ consists of uncountably many disjoint curves in $\mathbb{C}$. More precisely, every path-connected component of this set is an injective curve $\gamma:(0, \infty) \rightarrow \mathbb{C}$ or $\gamma:[0, \infty) \rightarrow \mathbb{C}$ with $\lim _{t \rightarrow \infty} \gamma(t)=\infty$.

This result is of interest not only in its own right, but provides an important ingredient in the dynamical study of the family of complex exponential maps. In this article, we will parametrize this family as

$$
E_{\kappa}: \mathbb{C} \rightarrow \mathbb{C} ; z \mapsto \exp (z)+\kappa
$$

Received by the editors September 1, 2005 and, in revised form, January 16, 2007.

2000 Mathematics Subject Classification. Primary 37F10; Secondary 30D05.

The first author was supported in part by a European fellowship of the Marie Curie Fellowship Association.

The second author was supported in part by a postdoctoral fellowship of the German Academic Exchange Service (DAAD) and by the German-Israeli Foundation for Scientific Research and Development (G.I.F.), grant no. G-643-117.6/1999. 
rather than $\exp (\lambda z)$ as above ${ }^{1}$ (note that the two maps are conformally conjugate to each other if $\lambda=\exp (\kappa))$. Theorem 1.1 will thus be proved by showing the corresponding statement for the set

$$
\mathcal{I}:=\left\{\kappa \in \mathbb{C}: E_{\kappa}^{n}(\kappa) \rightarrow \infty\right\}
$$

of escaping parameters.

This set is an analog to the exterior of the Mandelbrot set $\mathcal{M}$ for quadratic polynomials. The foliation of the open set $\mathbb{C} \backslash \mathcal{M}$ by parameter rays introduced by Douady and Hubbard $[\mathrm{DH}]$ has played a vital role in the understanding of quadratic polynomials. Although $\mathcal{I}$ is not an open set (in fact, it is nowhere dense), it has long been known to contain curves which can play the role of parameter rays (compare the historical remarks below). We will in fact provide a complete classification of escaping parameters in terms of these rays (Corollary 3.2): every escaping parameter is either on a parameter ray or the endpoint of such a ray. This is analogous to (and builds on) a similar classification [SZ1] obtained for the set of escaping points,

$$
I\left(E_{\kappa}\right):=\left\{z \in \mathbb{C}: E_{\kappa}^{n}(z) \rightarrow \infty \text { as } n \rightarrow \infty\right\},
$$

of an arbitrary exponential map $E_{\kappa}$, in terms of dynamic rays.

Our description of the set $\mathcal{I}$ also shows that the "Dimension Paradox" discovered in $[\mathrm{K}]$ occurs in the parameter plane as well. Indeed, Qiu [Q] showed that $\mathcal{I}$ has Hausdorff dimension 2. On the other hand, the set of parameter rays (without endpoints) only has Hausdorff dimension 1 [BBS]. Thus $\mathcal{I}$ contains a two-dimensional set of endpoints, each connected to $\infty$ by a curve in $\mathcal{I}$, such that all curves are disjoint and the union of these curves has Hausdorff dimension one.

Historical remarks. Parameter space of exponential maps was first studied in [BR, EL1], though the focus of these articles lay on the structure of hyperbolic components. A construction of (tails of) certain parameter rays consisting of escaping parameters was first sketched in [D], and carried out in [DGH]. (Compare the remark after Proposition 2.2.) In [S], full parameter rays were constructed for arbitrary bounded external addresses and in [F, FS], this was generalized to all parameter rays.

Classically, both dynamical and parameter rays were often referred to as "hairs" in the transcendental setting. We chose our terminology to emphasize the analogy with the polynomial case. Similarly, we chose the term external address, introduced in [SZ1], over that of itinerary. On the one hand, this highlights the relation to external angles of polynomials; on the other, it avoids the overloading of the latter term, which is frequently used for another kind of symbolic sequence both in exponential and polynomial dynamics (compare e.g. [SZ2, Section 4.1]).

\section{Preliminaries}

For the remainder of this paper, we will fix the function

$$
F:[0, \infty) \rightarrow[0, \infty) ; t \mapsto \exp (t)-1
$$

as a model for exponential growth. Following [SZ1], a sequence $\underline{s}=s_{1} s_{2} s_{3} \ldots$ of integers is called an external address; we also define the shift map on external addresses by $\sigma\left(s_{1} s_{2} s_{3} \ldots\right):=s_{2} s_{3} \ldots$

\footnotetext{
${ }^{1}$ We prefer our parametrization since it affords all maps the same asymptotic behavior near infinity.
} 

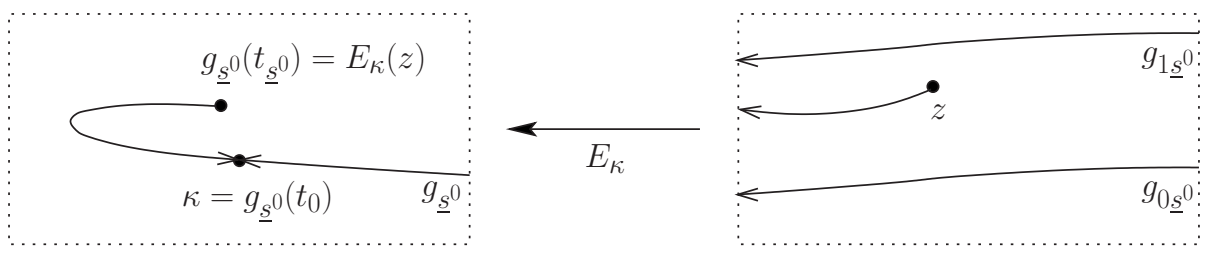

FiguRe 1. An illustration of the second part of Proposition 2.1. Here we have $\kappa \in g_{\underline{s}^{0}}$ for a fast address $\underline{s}^{0}$. Hence, its preimage rays (at addresses $k \underline{s}^{0}, k \in \mathbb{Z}$ ) end prematurely, and the preimage $z$ of $g_{\underline{s}^{0}}\left(t_{\underline{s}^{0}}\right)$ is not itself associated to any dynamic ray.

If $E_{\kappa}$ is an exponential map and $z \in \mathbb{C}$, then we say that $z$ has external address $\underline{s}$ if $\operatorname{Im} E_{\kappa}^{k-1}(z) \in\left(\left(2 s_{k}-1\right) \pi,\left(2 s_{k}+1\right) \pi\right)$ for all $k \geq 1$. An external address $\underline{s}$ is called exponentially bounded if there is an $x>0$ such that $2 \pi\left|s_{k}\right|<F^{k-1}(x)$ for all $k \geq 1$; the set of all such addresses is denoted by $\mathcal{S}_{0}$. An address $\underline{s} \in \mathcal{S}_{0}$ is called slow if there exists a subsequence $\sigma^{n_{k}}(\underline{s})$ of the $\sigma$-orbit of $\underline{s}$ such that all $\sigma^{n_{k}}(\underline{s})$ are exponentially bounded with the same $x$. Otherwise, $\underline{s}$ is called fast. For any external address $\underline{s}$, let us also define $t_{\underline{s}}:=\limsup _{k \rightarrow \infty} F^{-(\bar{k}-1)}\left(2 \pi\left|s_{k}\right|\right)$, where $F^{-(k-1)}$ denotes the $(k-1)$-th iterate of the function $F^{-1}:[0, \infty) \rightarrow[0, \infty)$. Note that $\mathcal{S}_{0}=\left\{\underline{s} \in \mathbb{Z}^{\mathbb{N}}: t_{\underline{s}}<\infty\right\}$.

2.1. Proposition (Dynamic rays [SZ1, Corollary 6.9]). Let $\kappa \in \mathbb{C}$ with $\kappa \notin I\left(E_{\kappa}\right)$. For every $\underline{s} \in \mathcal{S}_{0}$, there exists a unique simple curve $g_{\underline{s}}=g_{\underline{s}}^{\kappa}: X_{\underline{s}} \rightarrow I\left(E_{\kappa}\right)$, where $X_{\underline{s}}:=\left(t_{\underline{s}}, \infty\right)$ if $\underline{s}$ is slow and $X_{\underline{s}}:=\left[t_{\underline{s}}, \infty\right)$ if $\underline{s}$ is fast, such that

- $g_{\underline{s}}^{\kappa}(t)$ has external address $\underline{s}$ for all sufficiently large $t$ and

- for all $t>t_{\underline{s}}$, we have $\operatorname{Re} E_{\kappa}^{k}\left(g_{\underline{s}}^{\kappa}(t)\right)=F^{k}(t)+o(1)$ as $k \rightarrow \infty$.

For every $z \in I\left(E_{\kappa}\right)$, there exist unique $\underline{s}$ and $t$ such that $z=g_{\underline{s}}^{\kappa}(t)$. Furthermore, $E_{\kappa}\left(g_{\underline{s}}(t)\right)=g_{\sigma(\underline{s})}(F(t))$ for all $\underline{s}$ and $t$.

If $\kappa \in I\left(E_{\kappa}\right)$, then the above is still true with the modification that there are countably many addresses s such that $g_{\underline{s}}$ is not defined on the complete interval $X_{\underline{s}}$. More precisely, there exist some $\underline{s}^{0} \in \overline{\mathcal{S}}_{0}$ and $t^{0}$ such that $\kappa=g_{\underline{s}^{0}}^{\kappa}\left(t_{0}\right)$. If $\underline{s}$ is an address with $\sigma^{n}(\underline{s})=\underline{s}^{0}$, where $n \geq 1$, then $g_{\underline{s}}^{\kappa}(t)$ is not defined for $t \leq F^{-n}\left(t_{0}\right)$. If $z \in I\left(E_{\kappa}\right)$, then either $z=g_{\underline{s}}(t)$ for some $\underline{s} \in \mathcal{S}_{0}$ and $t \in X_{\underline{s}}$ (in which case $\underline{s}$ and $t$ are unique), or $E_{\kappa}^{n}(z)=g_{\underline{s}^{0}}(t)$ for some $n \geq 1$ and $t \leq t_{0}$.

The dynamic ray at address $\underline{s}$ is defined to be the curve $g_{\underline{s}}^{\kappa}:\left(t^{*}, \infty\right) \rightarrow \mathbb{C}$ for the least possible value of $t^{*}$. (This will be $t^{*}=t_{\underline{s}}$, unless $\kappa \in I\left(E_{\kappa}\right)$ and $\underline{s}$ is one of the countably many exceptional addresses.) If $t^{*}=t_{\underline{s}}$ and $\underline{s}$ is fast, then $g_{\underline{s}}^{\kappa}\left(t_{\underline{s}}\right)$ is called the endpoint of the ray $g_{\underline{s}}^{\kappa}$.

Remark 1. See Figure 1 for an illustration of the last part of the proposition, in the case where $\underline{s}^{0}$ is fast. The case where $\underline{s}^{0}$ is slow, but not periodic, is similar (except that the ray may not have a landing point in this case). If $\underline{s}^{0}$ is periodic, say of period $n$, then $g_{\underline{s}^{0}}$ is itself defined only for $t \geq F^{-n}\left(t_{0}\right)$, and every iterated preimage component of $g_{\underline{s}^{0}}\left(\left(F^{-n}\left(t_{0}\right), t_{0}\right)\right)$ is an arc tending to infinity in both directions (compare [R3, Figure 2]).

Remark 2. We say that the ray $g_{\underline{s}}:\left(t^{*}, \infty\right) \rightarrow \mathbb{C}$ lands at $z \in \hat{\mathbb{C}}$ if $z=\lim _{t \rightarrow t^{*}}\left(g_{\underline{s}}(t)\right)$. Proposition 2.1 shows that every dynamic ray at a fast address lands at an escaping 
point, but does not make any statements about the accumulation behavior of rays at slow addresses. Although it is true that many rays at slow addresses also land (compare [SZ1, Proposition 6.11] and [R2, Theorem 1]), this problem is much more complicated in general. Even for postsingularly finite exponential maps, there are dynamic rays at bounded addresses which do not land; compare [R4].

Remark 3. Let us define a topological space $X:=\bigcup_{\underline{s} \in \mathcal{S}_{0}}\left(\{\underline{s}\} \times X_{\underline{s}}\right)$ (that is, $X$ is the disjoint union of all $\left.X_{\underline{s}}\right) . X$ is endowed with the product topology of $\mathcal{S}_{0} \times[0, \infty)$, where $\mathcal{S}_{0}$ is understood as a discrete space and $[0, \infty)$ comes with the usual topology. Then the first part of the proposition implies that for every $\kappa \in \mathbb{C} \backslash \mathcal{I}$, there is a continuous bijection $\mathfrak{g}^{\kappa}: X \rightarrow I\left(E_{\kappa}\right)$ given by $\mathfrak{g}^{\kappa}(\underline{s}, t)=g_{\underline{s}}^{\kappa}(t)$.

The topology on $X$ is much finer than the planar topology on $I\left(E_{\kappa}\right)$, and it is impossible to embed $X$ in the plane. Thus $\mathfrak{g}$ can never be a homeomorphism. However, it is possible [R3] to endow $X$ with a different topology ${ }^{2}$ - turning it into a straight brush in the sense of $[\mathrm{AO}]$ - such that $\mathfrak{g}$ is a homeomorphism when restricted to large subsets of $X$ (compare the proof of Corollary 4.3). When the singular value $\kappa$ belongs to the Fatou set, then in fact $I\left(E_{\kappa}\right)$ is a straight brush [BD], and the map $\mathfrak{g}$ is a homeomorphism between the straight brush $X$ and $I\left(E_{\kappa}\right)$ [R3]. It seems reasonable to expect that $I\left(E_{\kappa}\right)$ is not a straight brush whenever $\kappa$ belongs to the Julia set; indeed, for a large class of such parameters it is known that there are dynamic rays which do not land and whose accumulation sets contain escaping points [R4].

Let us now turn to the question of how escaping parameters are organized in the parameter plane. By the above result, for every such parameter $\kappa$ there exist unique $\underline{s}$ and $t$ such that $\kappa=g_{\underline{s}}^{\kappa}(t)$. The set of parameters for which $t>t_{\underline{s}}$ - i.e. for which the singular value is not the endpoint of a dynamic ray — is described by the following result.

2.2. Proposition (Parameter rays $[\mathrm{F}, \mathrm{FS}]$ ). Let $\underline{s} \in \mathcal{S}_{0}$. Then for every $t>t_{\underline{s}}$, there exists a unique parameter $\kappa=: G_{\underline{s}}(t)$ with $\kappa=g_{\underline{s}}^{\kappa}(t)$. Furthermore, $G_{\underline{s}}$ : $\left(t_{\underline{s}}, \infty\right) \rightarrow \mathbb{C}$ is continuous, and $\left|G_{\underline{s}}(t)-\left(t+2 \pi i s_{1}\right)\right| \rightarrow 0$ as $t \rightarrow \infty$.

The curves $G_{\underline{s}}:\left(t_{\underline{s}}, \infty\right) \rightarrow \mathbb{C}$ are called parameter rays. As usual, the ray $G_{\underline{s}}$ is said to land at a point $\kappa \in \mathbb{C}$ if $G_{\underline{s}}(t) \rightarrow \kappa$ as $t \searrow t_{\underline{s}}$.

Remark 1. Proposition 2.2 was proved in [DGH] for the case of those bounded external addresses which are "regular". (With our notation, these are exactly those addresses satisfying $s_{k} \neq s_{1}$ for $k>1$.) Also, for every bounded address $\underline{s}$, a tail of the parameter ray $G_{\underline{s}}$ was constructed; that is, the existence of a curve $G_{\underline{s}}:\left(t_{\underline{s}}^{\prime}, \infty\right) \rightarrow \mathbb{C}$ was proved for sufficiently large $t_{\underline{s}}^{\prime} \geq t_{\underline{s}}$.

Remark 2. For parameter rays at regular bounded addresses, [DGH] also proves the existence of (nonescaping) landing points. In the present article, we are studying only the structure of the set $\mathcal{I}$ of escaping parameters, and are hence not making any claims regarding the landing of parameter rays at general slow addresses, which is a much more difficult problem. Indeed, one can ask whether it is true that every

\footnotetext{
${ }^{2}$ It is useful to think of this new topology as induced by the product topology of the lexicographic order on $\mathcal{S}_{0}$ and the usual topology on the real line. However, this is not quite accurate with our definitions: while it yields the correct topology in the $\underline{s}$-direction, we would need to change the parametrization of dynamic rays to obtain the right notion of convergence of potentials. See [R3] for details.
} 
parameter ray lands, and that every point in $\overline{\mathcal{I}}$ (the bifurcation locus) is either on a ray or a landing point of a ray. This is an analog of the celebrated and unresolved $M L C$ conjecture for quadratic polynomials, and would imply density of hyperbolicity in exponential parameter space by [RS2, Corollary A.5].

In order to extend Proposition 2.2 to escaping endpoints, we shall need two ingredients. The first is the fact that escaping endpoints depend holomorphically on the parameter.

2.3. Proposition (Holomorphic dependence [R3, Corollary 4.4]). Let $\underline{s}$ be a fast external address, and suppose that $\kappa$ is a parameter with $\kappa \notin g_{\sigma^{n}(\underline{s})}^{\kappa}\left(\left[t_{\sigma^{n}(s)}, \infty\right)\right)$ for all $n \geq 1$. Then there exists a neighborhood $U$ of $\kappa$ such that the function

$$
U \times\left[t_{\underline{s}}, \infty\right) \rightarrow \mathbb{C},(\kappa, t) \mapsto g_{\underline{s}}^{\kappa}(t)
$$

is defined, continuous in both variables and holomorphic in $\kappa$ for fixed $t$.

Remark. In [R3], a different parametrization of $g_{\underline{s}}^{\kappa}$ was used. This is not essential because the reparametrization which occurs is independent of $\kappa$.

We also require the following "Squeezing Lemma" which was proved in [RS1].

2.4. Proposition $([\mathrm{RS} 1$, Theorem 3.5$])$. Let $\gamma:[0, \infty) \rightarrow \mathbb{C}$ be a curve in parameter space such that for all $t$, all periodic points of $E_{\gamma(t)}$ are repelling. If furthermore $\lim _{t \rightarrow \infty}|\gamma(t)|=\infty$, then $\gamma$ contains the curve $G_{\underline{s}}\left(\left[t_{0}, \infty\right)\right)$ for some $\underline{s} \in \mathcal{S}_{0}$ and $t_{0}>t_{\underline{s}}$.

\section{Classification of escaping Parameters}

We are now ready to give a classification of those parameters for which the singular value is the escaping endpoint of a dynamic ray.

3.1. Theorem. Let $\underline{s} \in \mathcal{S}_{0}$ be fast. Then there is a unique parameter $\kappa$ with $\kappa=g_{\underline{s}}^{\kappa}\left(t_{\underline{s}}\right)$. Furthermore, the parameter ray $G_{\underline{s}}$ lands at this parameter $\kappa$.

Proof. Let $L_{\underline{s}}$ denote the limit set of the parameter ray $G_{\underline{s}}$ as $t \searrow t_{\underline{s}}$, and let $A_{\underline{s}}$ consist of all parameters $\kappa \in \mathbb{C}$ with $g_{\underline{s}}^{\kappa}\left(t_{\underline{s}}\right)=\kappa$. We need to show that $L_{\underline{s}}=A_{\underline{s}}$ and that this set consists of a single parameter.

In view of the results cited in the previous section, the idea of the proof is relatively straightforward. By continuity of $g_{\underline{s}}^{\kappa}(t)$ in $\kappa$ and $t$, we ought to have $L_{\underline{s}} \subset A_{\underline{s}}$; as the zero set of an analytic function, $A_{\underline{s}}$ is discrete. Hence $L_{\underline{s}}$ consists of a single point, and $A_{\underline{s}} \subset L_{\underline{s}}$ follows from Hurwitz's theorem. However, a little bit of care is required to carry this idea through, as e.g. it is a priori conceivable that $L_{\underline{s}}$ contains parameters for which $g_{\underline{s}}^{\kappa}\left(t_{\underline{s}}\right)$ is not defined.

Claim 1. $A_{\underline{s}}$ is closed and contains $L_{\underline{s}}$.

Proof. Consider the set

$$
B_{\underline{s}}:=\bigcap_{t_{0}>t_{\underline{s}}} \overline{\left\{\kappa \in \mathbb{C}: \exists t \in\left[t_{\underline{s}}, t_{0}\right]: \kappa=g_{\underline{s}}^{\kappa}(t)\right\}}=\overline{A_{\underline{s}}} \cup \bigcap_{t_{0}>t_{\underline{s}}} \overline{G_{\underline{s}}\left(\left(t_{\underline{s}}, t_{0}\right]\right)}=\overline{A_{\underline{s}}} \cup L_{\underline{s}} .
$$

To prove Claim 1, we show that $B_{\underline{s}} \subset A_{\underline{s}}$. So let $\kappa \in B_{\underline{s}}$ and choose sequences $\kappa_{j} \rightarrow \kappa$ and $t_{j} \rightarrow t_{\underline{s}}$ with $g_{\underline{s}}^{\kappa_{j}}\left(t_{j}\right)=\kappa_{j}$. Let $n \geq 0$ be the smallest number for which 
$g_{\sigma^{n}(\underline{s})}^{\kappa}\left(F^{n}\left(t_{\underline{s}}\right)\right)$ is defined (such an $n$ exists by Proposition 2.1). By Proposition 2.3,

$$
\begin{aligned}
g_{\sigma^{n}(\underline{s})}^{\kappa}\left(F^{n}\left(t_{\underline{s}}\right)\right) & =\lim _{j \rightarrow \infty} g_{\sigma^{n}(\underline{s})}^{\kappa_{j}}\left(F^{n}\left(t_{j}\right)\right) \\
& =\lim _{j \rightarrow \infty} E_{\kappa_{j}}^{n}\left(g_{\underline{s}}^{\kappa_{j}}\left(t_{j}\right)\right)=\lim _{j \rightarrow \infty} E_{\kappa_{j}}^{n}\left(\kappa_{j}\right)=E_{\kappa}^{n}(\kappa) .
\end{aligned}
$$

We claim that $n=0$. Suppose not. By minimality of $n$, it follows that the curve $g_{\sigma^{n}(\underline{s})}^{\kappa}$ contains $\kappa$, say $\kappa=g_{\sigma^{n}(\underline{s})}^{\kappa}(t)$ for some $t \geq F^{n}\left(t_{\underline{s}}\right)$. Since $\kappa \neq E_{\kappa}^{n}(\kappa)$, it follows that $t>F^{n}\left(t_{\underline{s}}\right)$. Now

$$
g_{\sigma^{2 n}(\underline{s})}^{\kappa}\left(F^{n}(t)\right)=E_{\kappa}^{n}(\kappa)=g_{\sigma^{n}(\underline{s})}^{\kappa}\left(F^{n}\left(t_{\underline{s}}\right)\right),
$$

which contradicts the statement on uniqueness of address and potential in Proposition 2.1. So $n=0$, and (2) reads $g_{\underline{s}}^{\kappa}\left(t_{\underline{s}}\right)=\kappa$ as claimed.

Claim 2. $A_{\underline{s}}$ is discrete.

Proof. Suppose, by contradiction, that $A_{\underline{s}}$ has an accumulation point $z \in \mathbb{C}$; then $z \in A_{\underline{s}}$ by Claim 1. Now $A_{\underline{s}}$ is the zero set of the function $\kappa \mapsto g_{\underline{s}}^{\kappa}\left(t_{\underline{s}}\right)-\kappa$. By Proposition 2.3, this function is analytic and its domain of definition $D$ is open. Let $U$ be the connected component of $D$ containing $z$. Then $U \subset A_{\underline{s}} \subset D$ by the identity theorem. Thus $U$ is a connected component both of the open set $D$ and the closed set $A_{\underline{s}}$, and therefore $U=D=A_{\underline{s}}=\mathbb{C}$. This is a contradiction as $\mathbb{C} \backslash A_{\underline{s}}$ is clearly nonempty.

Since the limit set $L_{\underline{s}} \subset A_{\underline{s}}$ is connected and $A_{\underline{s}}$ is discrete, $L_{\underline{s}}$ is either empty or consists of a single point. In the former case, the ray $G_{\underline{s}}$ would land at infinity, which is impossible by the Squeezing Lemma. Thus $G_{\underline{s}}$ lands at some point in $A_{\underline{s}} \subset \mathbb{C}$.

It remains to show that $A_{\underline{s}} \subset L_{\underline{s}}$. Let $\kappa_{0} \in A_{\underline{s}}$, and let $V$ be any small disk neighborhood of $\kappa_{0}$ with $\bar{V} \cap A_{\underline{s}}=\left\{\kappa_{0}\right\}$. If $t>t_{\underline{s}}$ is small enough, then by Hurwitz's theorem the function $\kappa \mapsto g_{\underline{s}}^{\kappa}(\bar{t})-\kappa$ has at least one zero in $V$; i.e. $G_{\underline{s}}(t) \in V$.

Remark. In a similar way, the Squeezing Lemma can be used to give an alternative proof of Proposition 2.2; see [R1, Section 5.12].

Together with Propositions 2.2 and 2.1, the previous theorem yields the desired classification of all escaping parameters. (Recall that $X_{\underline{s}}=\left[t_{\underline{s}}, \infty\right)$ when $\underline{s}$ is a fast external address, and $X_{\underline{s}}=\left(t_{\underline{s}}, \infty\right)$ when $\underline{s}$ is slow.)

3.2. Corollary. Let $\underline{s} \in \mathcal{S}_{0}$, and let $t \in X_{\underline{s}}$. Then there exists a unique parameter $\kappa=: G_{\underline{s}}(t)$ with $\kappa=g_{\underline{s}}^{\kappa}(t)$. Furthermore, $\bar{G}_{\underline{s}}$ is continuous in $t$; in particular, if $\underline{s}$ is fast, then $G_{\underline{s}}$ lands at an escaping parameter.

Conversely, for any escaping parameter $\kappa$, there exist unique $\underline{s} \in \mathcal{S}_{0}$ and $t \in X_{\underline{s}}$ such that $\kappa=G_{\underline{s}}(t)$.

Remark. With the notation introduced after Proposition 2.1, we can reformulate Corollary 3.2 as follows: there exists a continuous bijection $\mathcal{G}: X \rightarrow \mathcal{I}$ such that, for all $x \in X$, the parameter $\kappa:=\mathcal{G}(x)$ satisfies $\mathfrak{g}^{\kappa}(x)=\kappa$.

3.3. Corollary (First entry of external address). Let $\kappa_{0}$ be an escaping parameter, say $\kappa_{0}=g_{\underline{s}}^{\kappa_{0}}(t)$. Then $\operatorname{Im} \kappa_{0} \in\left(\left(2 s_{1}-1\right) \pi,\left(2 s_{1}+1\right) \pi\right)$.

Proof. For sufficiently large $t, G_{\underline{s}}(t)$ belongs to the strip $\left(\left(2 s_{1}-1\right) \pi,\left(2 s_{1}+1\right) \pi\right)$ by Proposition 2.2. Since the lines $\{\operatorname{Im} \kappa=(2 k+1) \pi\}$ only contain attracting and parabolic parameters $[\mathrm{BR}]$, the entire parameter ray $G_{\underline{s}}$ belongs to this strip. 


\section{Path components of sets obtained as a union of Curves}

To complete the proof of Theorem 1.1, we still need to show that the sets $G_{\underline{s}}\left(X_{\underline{s}}\right)$ indeed form the path-connected components of $\mathcal{I}$. To show this fact, we require a general topological principle (Proposition 4.2), which will be proved in this section. For completeness, we also apply this principle to show that every dynamic ray (possibly with endpoint) of an exponential map is a path-connected component of the set of escaping points, as stated in [SZ1] without proof.

We shall require the following basic fact of continuum theory.

4.1. Proposition. Let $K$ be a continuum, and let $\left(A_{n}\right)$ be an increasing sequence of nonempty closed subsets of $K$ such that $K=\bigcup A_{n}$. If every connected component of $A_{n}$ is also a connected component of $A_{n+1}$, then $A_{n}=K$ for all $n$.

Proof. We will apply this result only when $K=[0,1]$; let us restrict ourselves to this case for simplicity. (The proof in the general case is similar; for details, see [N, Theorem 5.16].) If $A_{n}=K$ for any $n$, then by assumption $A_{n}=K$ for all $n$. So suppose by contradiction that $A_{n} \neq K$ for all $n$. We will inductively define a nested sequence $\left(K_{j}\right)_{j \geq 0}$ of nonempty compact intervals such that $K_{j} \not \subset A_{k}$ for all $k$ and such that $K_{j+1} \subset K_{j} \backslash A_{j+1}$. This yields a contradiction since the intersection of all $K_{j}$ is nonempty, but is also disjoint from all $A_{j}$.

We begin the construction by setting $K_{0}:=K$. To construct $K_{j+1}$ from $K_{j}$, pick some point $x \in K_{j} \backslash A_{j+1}$ (this is possible by the induction hypothesis). Then there is some $n>j$ such that $x \in A_{n}$, and the connected component $C$ of $A_{n}$ containing $x$ is disjoint from $A_{j+1}$ by hypothesis. Since $C$ is compact, we can find a compact interval $K_{j+1}$ with $C \subsetneq K_{j+1} \subset K_{j} \backslash A_{j+1}$. For every $k \in \mathbb{N}$, either $C \not \subset A_{k}$ or $C$ is a connected component of $A_{k}$. In either case, $K_{j+1} \not \subset A_{k}$, as required.

4.2. Proposition. Let I be a Hausdorff topological space. Let $\Gamma$ be a partition of $I$ into path-connected subsets such that no union of two different elements of $I$ is path-connected.

Suppose that I can be written as a countable union of closed subsets $I_{k}$ such that

(a) $I_{k} \subset I_{k+1}$,

(b) every path-connected component of $I_{k}$ is contained in some element of $\Gamma$,

(c) every element of $\Gamma$ contains at most one path-connected component of $I_{k}$, and

(d) if $c \subset I$ is a simple closed curve which is completely contained in some element of $\Gamma$, then either $c \subset I_{k}$ or $c \cap I_{k}=\emptyset$.

Then $\Gamma$ is the set of path-connected components of $I$.

Remark. In the cases of interest to us, no element of $\Gamma$ will contain a simple closed curve, so (d) is automatically satisfied.

Proof. Since $I$ is Hausdorff, path-connectivity is the same as arc-connectivity. Let $\alpha:[0,1] \rightarrow I$ be any arc; we must show that $I$ is contained in a single element of $\Gamma$.

For every $t \in[0,1]$, the point $\alpha(t)$ belongs to some $\gamma=\gamma_{t} \in \Gamma$; let us denote by $J(t)$ the component of $\alpha^{-1}\left(\gamma_{t}\right)$ containing $t$. For every $k$, we also set $J_{k}^{\prime}:=\alpha^{-1}\left(I_{k}\right)$ (note that $J_{k}^{\prime}$ is compact), and

$$
J_{k}:=\bigcup_{t \in J_{k}^{\prime}} J(t) .
$$


Our goal is to show that the hypotheses of Proposition 4.1 apply to $K=[0,1]$ and $A_{k}=J_{k}$. To do so, we need to check a number of simple properties.

Claim 1. For every $t \in[0,1]$, the set $J(t)$ is compact.

Proof. $J(t)$ is an interval; let $a$ and $b$ be the endpoints of $J(t)$. Then $\gamma_{t} \cup \gamma_{a}$ is path-connected, since it contains the curve $\alpha([a, b))$ containing $\alpha(a)$ and $\alpha(t)$. By the assumption on $\Gamma$, this implies $\gamma_{t}=\gamma_{a}$, and thus $a \in J(t)$. Analogously, $b \in J(t)$.

Claim 2. Let $t \in J_{k}^{\prime}$. Then $J(t)$ contains exactly one component of $J_{k}^{\prime}$.

Proof. Otherwise, there is some interval $(a, b) \subset J(t) \backslash J_{k}^{\prime}$ with $a, b \in J_{k}^{\prime}$. Since $\alpha(a), \alpha(b) \in \gamma_{t}$, it follows by (c) that they belong to the same path-connected component of $I_{k}$. Let $\beta \subset I_{k}$ be an arc connecting $\alpha(a)$ and $\alpha(b)$. By (b), $\beta \subset \gamma_{a}$. Thus $\beta \cup \alpha((a, b)) \subset \gamma_{a}$ is a simple closed curve which intersects $I_{k}$ but is not contained in $I_{k}$, contradicting $(\mathrm{d})$.

Claim 3. $J_{k}$ is compact, and, for every $t \in J_{k}, J(t)$ is a connected component of $J_{k}$.

Proof. Let $A$ be a component of $[0,1] \backslash J_{k}^{\prime} \supset[0,1] \backslash J_{k}$. To fix ideas, let us suppose that neither 0 nor 1 is an endpoint of this interval, so that $A=(a, b)$ with $\bar{A} \cap J_{k}^{\prime}=$ $\{a, b\}$. Then $A \backslash J_{k}=A \backslash(J(a) \cup J(b))$.

Since $J(a) \cup J(b)$ is compact by Claim $1, A \backslash J_{k}$ is open. Writing $[0,1] \backslash J_{k}=$ $\bigcup A \backslash J_{k}$, where the union is taken over all intervals $A$ as above, it follows that $J_{k}$ is compact. Furthermore, by Claim $2, b \notin J(a)$. Thus $J(a) \neq J(b)$, so $A \backslash J_{k} \neq \emptyset$. Thus any two components of $J_{k}^{\prime}$ are separated by a point of $[0,1] \backslash J_{k}$.

Now let $t \in J_{k}$, and consider some point $t_{1} \in J_{k} \backslash J(t)$. Then $J(t)$ and $J\left(t_{1}\right)$ each contain a connected component of $J_{k}^{\prime}$. These are separated by a point of $[0,1] \backslash J_{k}$, which consequently also separates $J(t)$ and $J\left(t_{1}\right)$. Thus $t$ and $t_{1}$ belong to different connected components of $J_{k}$. So $J(t)$ is the connected component of $J_{k}$ containing $t$, completing the proof of Claim 3.

Claim 3 implies that every connected component of $J_{k}$ is also a connected component of $J_{k+1}$. Thus we can apply Proposition 4.1 to see that $J_{k}=[0,1]$ for some $k$. (Note that we can assume without loss of generality that $J_{1} \neq \emptyset$.) By Claim 3, this means that $\alpha([0,1])=\alpha(J(0)) \subset \gamma_{0}$, as required.

4.3. Corollary. Let $\kappa \in \mathbb{C}$. Then every path connected component of $I\left(E_{\kappa}\right)$ is

(a) a dynamic ray at a slow address, or

(b) a dynamic ray at a fast address together with its escaping endpoint, or

(c) (if $\kappa \in I\left(E_{\kappa}\right)$ ) an iterated preimage component of the ray (possibly with endpoint) containing the singular value.

Remark 1. According to the remark after Proposition 2.1, every component $\gamma$ of the last type is a curve to $\infty$. More precisely, according to whether the address $\underline{s}^{0}$ associated to the singular orbit is fast, periodic or slow but not periodic, $\gamma$ is an arc with one finite escaping endpoint, an arc tending to infinity in both directions, or an injective curve to infinity (which may or may not land at a point $z \notin I\left(E_{\kappa}\right)$ ), respectively.

Remark 2. Note that, while different rays form different path-connected components of $I\left(E_{\kappa}\right)$, they may belong to the same path-connected component of the 
Julia set $J\left(E_{\kappa}\right)$ (which is the closure of $I\left(E_{\kappa}\right)$ ). Indeed, often $J\left(E_{\kappa}\right)$ will be the entire complex plane. Even if this is not the case, several rays may have a common (nonescaping) landing point, and thus belong to the same path-connected component of the Julia set.

Similarly, different rays may belong to the same connected (rather than pathconnected) component of $I\left(E_{\kappa}\right)$, e.g. in the postsingularly finite case [DJM].

Proof. Let $\Gamma$ be the set of all curves of the types (a) to (c) listed above, which (by Proposition 2.1) exhausts all of $I\left(E_{\kappa}\right)$. Then the union of two different elements of $\Gamma$ is never path-connected, and no element of $\Gamma$ contains a simple closed curve. (This could only fail if there was some slow address $\underline{s} \in \mathcal{S}_{0}$ for which $g_{\underline{s}}^{\kappa}$ lands at an escaping point; this is impossible by [SZ1, Corollary 6.9].)

In [R3, Theorems 4.2 and 4.3], it was shown that there exists a relatively closed subset $Y \subset I\left(E_{\kappa}\right)$ with the following properties:

(a) $E_{\kappa}(Y) \subset Y$,

(b) for all $z \in I\left(E_{\kappa}\right)$, there is an $n \geq 0$ with $E_{\kappa}^{n}(z) \in Y$, and

(c) every connected component $C$ of $Y$ is the "tail" of some dynamic ray $g_{\underline{s}}$, in the following sense: either $C=g_{\underline{s}}\left(X_{\underline{s}}\right)$ or $C=g_{\underline{s}}([t, \infty))$ for some $t>\bar{t}_{\underline{s}}$.

(In fact, the set $Y$ is a "straight brush" in the sense of Aarts and Oversteegen [AO].)

It follows easily from (c) that every path-connected component of $I_{n}:=E_{\kappa}^{-n}(Y)$ is contained in a single element of $\Gamma$, and that the intersection of $I_{n}$ with any element of $\Gamma$ is path-connected. Thus we can apply Proposition 4.2 to $\Gamma$ and the sequence $\left(I_{n}\right)$, which completes the proof.

4.4. Remark (Alternative definition of dynamic rays). By Corollary 4.3, the following simple definition of dynamic rays is equivalent to the original one:

Let $E_{\kappa}$ be an exponential map and suppose that the singular value $\kappa$ does not escape. A dynamic ray of $E_{\kappa}$ is a maximal injective curve $\gamma:(0, \infty) \rightarrow \mathbb{C}$ in the escaping set. ${ }^{3}$

\section{ACCumulation Behavior of PARAMEter Rays}

In the proof of Corollary 4.3, we used the fact that a dynamic ray at a slow address cannot land at an escaping point. To complete the proof of Theorem 1.1, we shall show the analogous statement in the parameter plane, which requires two more facts about parameter rays. The first of these is a result from [RS2]. There the structure of parameter space was exploited to show that any two parameter rays which accumulate at a common point must share the same "kneading sequence" (a combinatorial invariant), and in particular satisfy the following combinatorial restriction.

5.1. Proposition ([RS2, Corollary A.2]). Suppose that $\underline{s}^{1}, \underline{s}^{2} \in \mathcal{S}_{0}$ such that $G_{\underline{s}^{1}}$ and $G_{\underline{s}^{2}}$ have a common accumulation point. Then $\left|s_{k}^{1}-s_{k}^{2}\right| \leq 1$ for all $k \geq 1$.

Our second ingredient shows that a parameter ray cannot land at an escaping parameter which is not the endpoint of a parameter ray. (This is a special case of some results obtained in [R3] regarding the continuous dependence of parameter

\footnotetext{
${ }^{3}$ As defined here, the parametrization of $\gamma$ need not be related to potentials as used earlier; we include the parametrization over $(0, \infty)$ only in order to exclude the endpoint, if any, from the definition of dynamic rays.
} 
rays on their external addresses: every ray is locally uniformly approximated by other parameter rays, so that the endpoint is the only possible accessible point.)

5.2. Proposition ([R3, Corollary 11.3]). Let $\underline{s} \in \mathcal{S}_{0}$, and let $L_{\underline{s}}$ denote the accumulation set of $G_{\underline{s}}$. If $G_{\underline{\tilde{s}}}(t) \in L_{\underline{s}}$ for some $\underline{\tilde{s}} \in \mathcal{S}_{0}$ and $t>t_{\underline{\tilde{s}}}$, then $G_{\underline{\tilde{s}}}\left(\left(t_{\underline{\tilde{s}}}, t\right)\right) \subset$ $L_{\underline{s}}$.

5.3. Corollary. Suppose that $\underline{s}$ is a slow address. Then $G_{\underline{s}}$ does not land at an escaping parameter.

Proof. By Proposition 5.2, $G_{\underline{s}}$ cannot land at a parameter of the form $G_{\underline{\tilde{s}}}(t)$ with $t>t_{\underline{\tilde{s}}}$. So suppose, by contradiction, that $G_{\underline{s}}$ lands at $G_{\underline{\tilde{s}}}\left(t_{\underline{\tilde{s}}}\right)$ for some fast external address $\underline{\tilde{s}}$. Applying Proposition 5.1, we see that $\left|\widetilde{s}_{n}-s_{n}\right| \leq 1$ for all $n$. This is impossible since $\underline{s}$ is slow and $\underline{\tilde{s}}$ is fast.

5.4. Theorem. The path-connected components of $\mathcal{I}$ are exactly the sets $G_{\underline{s}}\left(X_{\underline{s}}\right)$ with $\underline{s} \in \mathcal{S}_{0}$.

Remark. This completes the proof of Theorem 1.1.

Proof. Set $\Gamma:=\left\{G_{\underline{s}}\left(X_{\underline{s}}\right): \underline{s} \in \mathcal{S}_{0}\right\}$; then the elements of $\Gamma$ are pairwise disjoint. Again, the union of two such elements is never path-connected, and no parameter ray contains a simple closed curve, by Corollary 5.3.

We again wish to apply Proposition 4.2 to see that $\Gamma$ is the set of path-connected components of $\mathcal{I}$. To do so, we will construct an exhaustion of $\mathcal{I}$ by a sequence of "straight brushes", similarly as in the dynamical plane. (Compare [R3, Theorem 11.5].) This is somewhat more complicated in parameter space, so let us begin by outlining the strategy. We define closed sets $\mathcal{I}_{n}^{\prime}$, each of which is homeomorphic to a subset of a straight brush. If $\mathcal{I}_{n}$ is the union of all unbounded components of $\mathcal{I}_{n}^{\prime}$, then $\mathcal{I}_{n}$ is a straight brush, each of whose components is contained in exactly one element of $\Gamma$. To complete the proof, it remains to show that the sets $\mathcal{I}_{n}$ also exhaust $\mathcal{I}$, which is essentially a compactness argument. The details follow.

We begin by defining, for each $n \in \mathbb{N}$,

$$
\mathcal{I}_{n}^{\prime}:=\left\{\kappa \in \mathcal{I}: \operatorname{Re} E_{\kappa}^{j}(\kappa) \geq K(\kappa) \text { for all } j \geq n\right\},
$$

where $K(\kappa):=\log ^{+}(|\kappa|)+10$. Clearly each $\mathcal{I}_{n}{ }^{\prime}$ is a closed subset of $\mathcal{I}$; furthermore $\mathcal{I}_{n}^{\prime} \subset \mathcal{I}_{n+1}^{\prime}$ and $\mathcal{I}=\bigcup \mathcal{I}_{n}^{\prime}$.

Let us endow the space $\widehat{X}:=X \cup\{\infty\}$ with the "straight brush" topology from [R3] (compare the remark after Proposition 2.1). As in the 'ray-wise discrete' topology we have considered so far, every connected component of $X$ is of the form $\{\underline{s}\} \times X_{\underline{s}}$, and every closed connected subset of $\widehat{X}$ is path-connected.

Set $\widehat{\mathcal{I}}:=\mathcal{I} \cup\{\infty\}$, and consider the map $\varphi: \widehat{\mathcal{I}} \rightarrow \widehat{X}$ which takes $\infty$ to $\infty$ and maps every parameter $\kappa \in \mathcal{I}$ to the unique pair $(\underline{s}, t) \in X$ with $\kappa=g_{\underline{s}}^{\kappa}(t)$. It follows from [R3, Corollary 4.4] that the map $\varphi$ is continuous on $\mathcal{I}_{n}^{\prime} \cup\{\infty\}$ for every $n .{ }^{4}$ So any connected set $C \subset \mathcal{I}_{n}^{\prime}$ is mapped into a connected component of $X$ under $\varphi$, and is thus a piece of a single parameter ray. If $C$ is unbounded, then $C$ is a tail of this ray (in the same sense as in the proof of Corollary 4.3).

\footnotetext{
${ }^{4}$ With the notation after Corollary $3.2, \varphi=\mathcal{G}^{-1}$. Using the bijectivity of $\varphi$, one can show that $\varphi$ is actually a homeomorphism when restricted to $\mathcal{I}_{n}^{\prime}$; however, both the definition of $\varphi$ and its continuity on the mentioned sets are independent of Corollary 3.2.
} 
Let $\widehat{\mathcal{I}}_{n}$ denote the component of $\mathcal{I}_{n}^{\prime} \cup\{\infty\}$ containing $\infty$; by the property of the topology of $\widehat{X}$ mentioned above, the set $\widehat{\mathcal{I}}_{n}$ is path-connected. We set $\mathcal{I}_{n}:=$ $\widehat{\mathcal{I}}_{n} \backslash\{\infty\}$. Then $\mathcal{I}_{n}$ is a closed subset of $\mathcal{I}$. Since $\widehat{\mathcal{I}}_{n}$ is path-connected, every connected component of $\mathcal{I}_{n}$ is unbounded, and is thus a tail of some parameter ray.

We can now apply Proposition 4.2 to see that the path-connected components of $\widetilde{\mathcal{I}}:=\bigcup \mathcal{I}_{n}$ are exactly the sets $\gamma \cap \widetilde{\mathcal{I}}$ with $\gamma \in \Gamma$. It remains to show that $\widetilde{\mathcal{I}}=\mathcal{I}$; i.e., that $G_{\underline{s}}(t) \in \tilde{\mathcal{I}}$ for all $\underline{s} \in \mathcal{S}_{0}$ and $t \in X_{\underline{s}}$. So let $\underline{s} \in \mathcal{S}_{0}$ and consider the (closed) sets

$$
\begin{aligned}
& A_{n}:=\left\{t \in X_{\underline{s}}: G_{\underline{s}}(t) \in \mathcal{I}_{n}^{\prime}\right\} \quad \text { and } \\
& B_{n}:=\left\{t \in X_{\underline{s}}: G_{\underline{s}}(t) \in \mathcal{I}_{n}\right\} .
\end{aligned}
$$

By [FS, Theorem 3.2], the set $B_{1}$ is nonempty. Note that $B_{n}$ is the unbounded component of $A_{n}$ for all $n$, and that $X_{\underline{s}}=\bigcup A_{n}$. We need to establish the stronger property that $X_{\underline{s}}=\bigcup B_{n}$, which will be achieved through a standard connectivity argument.

Claim. For every $T \in X_{\underline{s}}$, there exists $n \in \mathbb{N}$ such that $A_{n}$ is a neighborhood of $T$ in $X_{\underline{s}}$.

Proof. It follows easily from [R3, Theorem 4.2] that we can find a natural number $n=n(T)$ with the following property: if $\kappa$ is sufficiently close to $G_{\underline{s}}(T)$ and $t \in X_{\underline{s}}$ is sufficiently close to $T$, then

$$
\operatorname{Re} E_{\kappa}^{m}\left(g_{\underline{s}}^{\kappa}(t)\right)>K\left(G_{\underline{s}}(T)\right)+1
$$

for all $m \geq n$. Also, $K(\kappa)$ is continuous in $\kappa$, and $G_{\underline{s}}(t)$ is continuous in $t$. So if $t$ is close enough to $T$, then $\kappa:=G_{\underline{s}}(t)$ satisfies

$$
\operatorname{Re} E_{\kappa}^{m}(\kappa)=\operatorname{Re} E_{\kappa}^{m}\left(g_{\underline{s}}^{\kappa}(t)\right)>K\left(G_{\underline{s}}(T)\right)+1 \geq K(\kappa)
$$

for all $m \geq n$. Therefore $t \in A_{n}$, completing the proof of the Claim.

For every $t \in X_{\underline{s}}$, define

$B(t):=\bigcup\left\{C: C\right.$ is the connected component of $A_{n}$ containing $t$ for some $\left.n \in \mathbb{N}\right\}$.

By the Claim, $B(t)$ is open for every $t$, so the $B(t)$ form a partition of $X_{\underline{s}}$ into open sets. Since $X_{\underline{s}}$ is connected, this means that $B(t)=X_{\underline{s}}$ for all $t$. On the other hand, for $t_{0} \in B_{1}$ and $n \in \mathbb{N}, B_{n}$ is the connected component of $A_{n}$ containing $t_{0}$. Thus $\bigcup B_{n}=B\left(t_{0}\right)=X_{\underline{s}}$. This completes the proof.

\section{ACKNOWLEDGMENTS}

We would like to thank the referee for useful suggestions, and Vlad Vicol for an interesting historical discussion.

\section{References}

[AO] Jan M. Aarts and Lex G. Oversteegen, The geometry of Julia sets, Trans. Amer. Math. Soc. 338 (1993), no. 2, 897-918. MR1182980 (93j:30021)

[BBS] Mihai Bailesteanu, Horia Vlad Balan, and Dierk Schleicher, Hausdorff dimension of exponential parameter rays and their endpoints, arXiv:0704.3087, conditionally accepted for publication in Nonlinearity.

[BR] I. Noel Baker and Philip J. Rippon, Iteration of exponential functions, Ann. Acad. Sci. Fenn. Ser. A I Math. 9 (1984), 49-77. MR752391 (86d:58065) 
[BD] Ranjit Bhattacharjee and Robert L. Devaney, Tying hairs for structurally stable exponentials, Ergodic Theory Dynam. Systems 20 (2000), no. 6, 1603-1617. MR1804947 (2002a:37064)

[BDG1] Clara Bodelón, Robert L. Devaney, Michael Hayes, Gareth Roberts, Lisa R. Goldberg, and John H. Hubbard, Hairs for the complex exponential family, Internat. J. Bifur. Chaos Appl. Sci. Engrg. 9 (1999), no. 8, 1517-1534. MR1721835 (2001a:37055)

[BDG2] _ Dynamical convergence of polynomials to the exponential, J. Differ. Equations Appl. 6 (2000), no. 3, 275-307. MR1785056 (2001f:37055)

[D] Robert L. Devaney, Julia sets and bifurcation diagrams for exponential maps, Bull. Amer. Math. Soc. (N.S.) 11 (1984), no. 1, 167-171. MR741732 (86b:58091)

[DGH] Robert L. Devaney, Lisa R. Goldberg, and John H. Hubbard, A dynamical approximation to the exponential map by polynomials, Preprint, MSRI Berkeley, 1986, published as Bodelón, Devaney, Hayes, Roberts, Goldberg and Hubbard, 1999, 2000.

[DJM] Robert L. Devaney, Xavier Jarque, and Mónica Moreno Rocha, Indecomposable continua and Misiurewicz points in exponential dynamics, Internat. J. Bifur. Chaos Appl. Sci. Engrg. 15 (2005), no. 10, 3281-3293. MR2192642 (2006j:37053)

[DH] Adrien Douady and John Hubbard, Etude dynamique des polynômes complexes, Prépublications mathémathiques d'Orsay (1984 / 1985), no. 2/4.

[EL1] Alexandre È. Eremenko and Mikhail Yu. Lyubich, Iterates of entire functions, Preprint, Physico-Technical Institute of Low-Temperature Physics Kharkov, 1984. MR769199 (86c:30044)

[EL2] - Dynamical properties of some classes of entire functions, Ann. Inst. Fourier (Grenoble) 42 (1992), no. 4, 989-1020. MR1196102 (93k:30034)

[E] Leonhardt Euler, De formulis exponentialibus replicatis, Acta Acad. Petropolitanae 1 (1777), 38-60.

[F] Markus Förster, Parameter rays for the exponential family, Diplomarbeit, Techn. Univ. München, 2003, Available as Thesis 2003-03 on the Stony Brook Thesis Server.

[FS] Markus Förster and Dierk Schleicher, Parameter rays for the exponential family, Preprint, 2005, to appear in Ergodic Theory Dynam. Systems.

[HSS] John H. Hubbard, Dierk Schleicher, and Mitsuhiro Shishikura, A topological characterization of postsingularly finite exponential maps and limits of quadratic differentials, Manuscript, 2004, submitted for publication.

[K] Bogusława Karpińska, Hausdorff dimension of the hairs without endpoints for $\lambda \exp z$, C. R. Acad. Sci. Paris Sér. I Math. 328 (1999), no. 11, 1039-1044. MR1696203 (2000e:37054)

[LSV] Bastian Laubner, Dierk Schleicher, and Vlad Vicol, A combinatorial classification of postsingularly periodic complex exponential maps, Preprint, 2006, arXiv:math.DS/ 0602602, conditionally accepted for publication in Discrete Contin. Dynam. Syst.

[M] John Milnor, Dynamics in one complex variable, third ed., Annals of Mathematics Studies, vol. 160, Princeton University Press, Princeton, NJ, 2006. MR2193309 (2006g:37070)

[N] Sam B. Nadler, Jr., Continuum theory. An introduction, Monographs and Textbooks in Pure and Applied Mathematics, vol. 158, Marcel Dekker Inc., New York, 1992. MR1192552 (93m:54002)

[Q] Weiyuan Qiu, Hausdorff dimension of the $M$-set of $\lambda \exp (z)$, Acta Math. Sinica (N.S.) 10 (1994), no. 4, 362-368. MR1416147 (97g:30024)

[R1] Lasse Rempe, Dynamics of exponential maps, doctoral thesis, Christian-AlbrechtsUniversität Kiel, 2003, http://e-diss.uni-kiel.de/diss_781/.

[R2] - A landing theorem for periodic rays of exponential maps, Proc. Amer. Math. Soc 134 (2006), no. 9, 2639-2648, arXiv:math.DS/0307371. MR2213743

[R3] Topological dynamics of exponential maps on their escaping sets, Ergodic Theory Dynam. Systems 26 (2006), no. 6, 1939-1975, arXiv:math.DS/0309107. MR2279273

[R4] - Nonlanding dynamic rays of exponential maps, Ann. Acad. Sci. Fenn. Math. 32 (2007), no. 2, 353-369, arXiv:math.DS/0511588. MR2337482

[RS1] Lasse Rempe and Dierk Schleicher, Bifurcations in the space of exponential maps, Preprint \#2004/03, Institute for Mathematical Sciences, SUNY Stony Brook, 2004, arXiv:math.DS/0311480, submitted for publication.

[RS2] C Combinatorics of bifurcations in exponential parameter space, Transcendental Dynamics and Complex Analysis (P. Rippon and G. Stallard, eds.), London Math. Soc. Lecture Note Ser., Cambridge Univ. Press, 2007, pp. 317-370, arXiv:math.DS/0408011. 
[S] Dierk Schleicher, On the dynamics of iterated exponential maps, Habilitation thesis, TU München, 1999.

[SZ1] Dierk Schleicher and Johannes Zimmer, Escaping points of exponential maps, J. London Math. Soc. (2) 67 (2003), no. 2, 380-400. MR1956142 (2003k:37067)

[SZ2] - Periodic points and dynamic rays of exponential maps, Ann. Acad. Sci. Fenn. Math. 28 (2003), 327-354. MR1996442 (2004e:37068)

KPMG Deutsche Treuhand-Gesellschaft, Marie-Curie-Strasse 30, 60439 Frankfurt/ Main, Germany

E-mail address: mfoerster@kpmg.com

Department of Mathematical Sciences, University of Liverpool, Liverpool L69 7ZL, United Kingdom

E-mail address: 1.rempe@liverpool.ac.uk

International University Bremen, P.O. Box 750 561, 28725 Bremen, Germany

E-mail address: dierk@iu-bremen.de 